\title{
Solo en caso de ambigüedad o la coherencia en la enseñanza de la tilde
}

\author{
Elia Saneleuterio ${ }^{1}$ \\ Universitat de València, España
}

La Ortografía de la lengua española, en su última versión, dedica el apartado 3.4.3.3 del capítulo sobre la acentuación gráfica a unos vocablos que han estado en el punto de mira por este motivo durante los últimos años: el adverbio solo y los demostrativos femeninos y masculinos en función pronominal.

Allí, la Real Academia Española (RAE) y la Asociación de Academias de la Lengua Española (ASALE) se refieren a una tilde prescrita en algunas normativas anteriores, pero que ya hace un par de décadas que se presenta como injustificada. En efecto, estas palabras no deben llevarla según las reglas generales de acentuación, dado que son llanas acabadas en vocal o vocal más ese. Y tampoco cumplen las condiciones para ser consideradas como casos de tilde diacrítica. Por ello, desde 1999 se eliminó la tilde de estas voces, si bien se consideraba obligatoria solo en caso de ambigüedad (RAE 1999: 27). Al respecto establece la actual normativa lo siguiente:

Sin embargo, puesto que ese empleo tradicional de la tilde diacrítica no opone en estos casos formas tónicas a otras átonas formalmente idénticas (requisito prosódico que justifica el empleo de la tilde diacrítica), ya que tanto el adjetivo solo como los determinantes demostrativos son palabras

\footnotetext{
Para correspondencia, dirigirse a: Elia Saneleuterio (elia.saneleuterio@uv.es). Departamento de Didáctica de la Lengua y la Literatura en la Facultad de Magisterio de la Universitat de València. Avda. Els Tarongers, 4; 46022, Valencia (España). Tel.: 003496162 5353.
} 
tónicas, lo mismo que el adverbio solo y los pronombres demostrativos, a partir de ahora se podrá prescindir de la tilde en estas formas incluso en casos de doble interpretación. (RAE y ASALE 2010: 269)

Esta nota pretende comentar algunas inconsistencias como la citada, con el objetivo de aportar al respecto algunas indicaciones que ayuden a conseguir la plena coherencia en la enseñanza de la acentuación gráfica, por un lado, y por otro a dotar, a quien está en proceso de aprenderla o reforzarla, de estrategias y competencias estables a través del tiempo.

\section{EL ADVERBIO SOLO Y LOS PRONOMBRES DEMOSTRATIVOS MASCULINOS Y FEMENINOS}

Si abordamos en primer lugar la tilde de la palabra solo, que da título a esta nota, y hacemos un poco de historia, recordaremos sin ninguna dificultad que este término llevaba tilde cuando se refería al adverbio solo, al igual que sucedía con los pronombres demostrativos respecto de sus formas con función adjetiva, con la excepción del género neutro (esto) - que, por cierto, algunas personas tildan por hipercorrección-.

Es curioso que lo recordemos "sin ninguna dificultad" 2 , cuando hace diecinueve años que esta tilde está suprimida... o al menos era su obligatoriedad la que se eliminó en la Ortografía académica de 1999, si bien la frase normativa "Cuando quien escribe perciba riesgo de ambigüedad, llevará acento ortográfico en su uso adverbial" (RAE, 1999: 27) parecía implicar que el adverbio solo se debía tildar solo en caso de ambigüedad. De hecho, los estudiantes actuales -la mayoría de los cuales no había nacido todavía cuando se publicó esta penúltima reforma ortográfica- reciben hoy en día en demasiadas ocasiones instrucción que ni siquiera es acorde a las normas de $1999^{3}$, las cuales incluso están desfasadas desde que dicho

2 En realidad, no resulta tan extraño si tenemos en cuenta que, desde 2010, la polémica no ha cesado, y se cuentan por decenas los escritores y escritoras que, aún hoy, desde su posición de prestigio predican cierta desobediencia en este aspecto, casi como signo de identidad lingüística. A pesar de la existencia de tildistas apasionados, lo que predomina en el uso general es la ausencia de criterio o "salpimentar al gusto", tal y como demuestra el análisis de la prensa realizado por Gándara (2014).

3 Y no hablamos del uso prescriptivo de la tilde en las mayúsculas, siendo sorprendente que haya profesores que lo relativicen aludiendo a una licencia que nunca existió -si bien es 
alumnado tiene uso de razón -pensemos, por ejemplo, que quienes inician la secundaria ahora, en 2010 apenas contaban 4 años-.

Para mayor perplejidad, si nos remontamos todavía más en la historia, observamos que en la primera ortografía académica (RAE 1857) nada se dice del adverbio solo y el mismo Diccionario de autoridades no lo tilda hasta 1925 (RAE 1726-1739/1969). Una tilde que no ha estado vigente ni siquiera un siglo no parece contar con tanta tradición como para que su pérdida genere nostalgia (Álvarez 2017).

Por otro lado, observamos que en el texto normativo que regula actualmente su uso subyace todavía mayor ambigüedad. Si bien queda perfectamente explicada -ya se justificaba en 1999- la supresión de la tilde tanto en el adverbio solo como en los mencionados demostrativos, no entendemos por qué las academias no actúan con mayor contundencia.

Así pues, como primer apunte, cabe cuestionar la funcionalidad de la obligatoriedad cuando hay riesgo de ambigüedad, norma que se prescribía desde 1999 hasta 2010. En el Diccionario panhispánico de dudas se insiste en ello: "cuando esta palabra pueda interpretarse en un mismo enunciado como adverbio o como adjetivo, se utilizará obligatoriamente la tilde en el uso adverbial para evitar ambigüedades" (RAE y ASALE 2005: 639). Ello parecía indicar que siempre que encontráramos un solo sin tilde había que interpretarlo como adjetivo, excepto en caso de que la frase careciera de sentido y tuviera que tratarse necesariamente de un adverbio. Así expresada, esta idea se nos presenta como un despropósito; sin embargo, se trata de la traducción práctica de lo que expresaba la normativa de 1999. Lo cierto es que el uso no refrendó la norma, y pocos fueron quienes usaron la tilde respetando la recomendación académica. Así lo atestigua Frago Gracia:

[...] en la escritura de los particulares parece predominar aún la norma precedente, y en general también es lo que sucede en muchos periódicos españoles y americanos. Desde luego no he leído ninguno en el que haya un absoluto acatamiento a esta innovación, cuyo triunfo sin duda requerirá bastante tiempo, sobre todo si en la enseñanza escolar no se sigue con todo rigor. (Frago 2012: 35)

Siendo que jamás acabó de funcionar este criterio, no entendemos por qué en 2010, y luego en 2012 (RAE y ASALE, 2012: 54-55), se redacta en la

cierto que hay manuales de estilo de algunos periódicos (en España puede citarse, por ejemplo, el caso de El Mundo, desde su primera edición [1996]) que regulan su propia ortografía al margen de las academias-. 
misma línea el nuevo texto académico, y todavía entendemos menos que esa misma línea conlleve implicaciones diferentes. ¿Qué significa que se permite tildar incluso en casos de riesgo de ambigüedad? Yo entiendo que, tratándose de una frase donde el solo o el demostrativo sean adjetivos, la ambigüedad va a persistir... ¿Cómo sabemos si el autor decidió "prescindir de la tilde en estas formas incluso en casos de doble interpretación" (RAE y ASALE 2010: 269)?

Además, percibo una segunda implicación: ¿significa que podemos tildar el adverbio solo y los pronombres demostrativos femeninos y masculinos siempre que queramos, aun no habiendo riesgo de interpretarlos como adjetivos? En principio, la regulación académica no lo explicita, y no parece tampoco dejar esta posibilidad implícita, ni en la Ortografía de la lengua española (RAE y ASALE 2010) ni en el Diccionario de la lengua española (RAE y ASALE 2014). Empero, la opinión general -y también la experta- así lo interpreta. Podemos convenir, pues, que una regla tan anárquica carece de función.

En el fondo de esta cuestión subyace una especie de temor a la ambigüedad lingüística. Ahora que, con el beneplácito de la Asociación de Academias de la Lengua Española -no sabemos bien si contamos además con su recomendación- podemos olvidarnos de la tilde de estas palabras, ¿qué pasa realmente con esa ambigüedad? Veámoslo con algunos ejemplos.

\section{En la cocina solo soy incapaz de cocinar. Pvo}

En la cocina solo soy incapaz de cocinar.

Dado que el adjetivo solo y el adverbio solo realizan funciones diferentes, estas tienen colocaciones típicas que pueden ayudar a interpretar correctamente el mensaje: En la cocina soy incapaz de cocinar solo vs. En la cocina soy incapaz solo de cocinar ${ }^{4}$. Igual que podemos modificar la redacción, podemos emplear sinónimos o estructuras equivalentes cuyo significado resulte explícito: En la cocina únicamente soy incapaz de cocinar. Vemos aquí como el problema de la ambigüedad trasciende la tilde, que no deja de ser una marca gráfica. Ni siquiera estamos propiamente ante casos de doble interpretación, sino triple -múltiple en otros casos-, pues en el ejemplo, aun teniendo claro que aquí solo es un adjetivo, ¿a qué se

4 Otra cosa significaría Solo en la cocina soy incapaz de cocinar. 
refiere?: En la cocina sin compañia soy incapaz de cocinar y En la cocina sin ayuda soy incapaz de cocinar son soluciones posibles que evidencian la imposibilidad de cualquier lengua de garantizar enunciados inequívocos.

En caso de género femenino, siendo el adjetivo sola, obviamente la ambigüedad respecto del adverbio es inexistente, pero persiste la duda acerca del significado de esa 'soledad' predicada: En la cocina sola soy incapaz de cocinar. Cuando aparece el adverbio, la clave de interpretación puede quedar anclada en diversos elementos: En la cocina solo soy incapaz de cocinar [referente femenino del sujeto, que conduce a interpretar solo como adverbio]; Ella solo es incapaz de cocinar [sujeto femenino explícito, que obliga a su interpretación como adverbio]; Solo soy inepta para cocinar [adjetivo predicativo con variación de género, que obliga a interpretar solo como adverbio]...). Entre todas ellas, únicamente la primera puede ser ambigua por causa del solo en caso de no conocerse el referente; por ejemplo, en un enunciado escrito no suficientemente contextualizado.

De todas formas, la posibilidad de la anfibología es parte de la riqueza del lenguaje, participa de su misterio y de la fuerza con la que nos atrae a los filólogos y a quienes nos dedicamos a la enseñanza de la lengua. ¿Qué problema tiene Viajó solo a Finlandia? Hace veinte años no podíamos jugar con este enunciado... Hay quien piensa que es un paso atrás, mientras que otras personas estamos convencidas de que la coherencia en la ortografía es un signo de la madurez de una lengua; la ambigüedad existe en la oralidad, y la función de la escritura no es deshacer esas dobles interpretaciones. En efecto, propiamente, la tilde diacrítica no aclara ambigüedades, sino que ayuda a identificar un patrón fónico, dado que en el contexto de una frase oral no pronunciamos estas parejas con la misma fuerza prosódica -por ejemplo, el artículo es átono y él pronombre es tónico, a diferencia de la doble tonicidad de solo y de los demostrativos-.

\section{OTRAS INCOHERENCIAS EN EL USO PRESCRIPTIVO DE LA TILDE}

¿Y qué ocurre con otras soluciones de acentuación gráfica que prescribe la Ortografía de la lengua española de 2010? Veamos, solo como apunte, algunos casos que me parecen reseñables. En primer lugar, y como hemos estado viendo, queda bien definida la función de la tilde diacrítica: distingue palabras tónicas "de uso muy frecuente" de sus homógrafas átonas $-\mathrm{u}$ 
homógrafas excepto por la tilde, claro- (RAE y ASALE 2010: 230-231). Pero ¿qué ocurre con la parte de la definición que tiene que ver con el "uso frecuente"? Si dejamos los interrogativos y exclamativos, que constituyen un grupo cerrado homogéneo y coherente, y nos centramos en el listado de monosílabos con tilde diacrítica, ¿acaso el verbo dé es frecuente, o lo es el sustantivo té -y su plural, tés, raramente confundible con las letras tes-? Por no mencionar que se trata, respectivamente, de un sustantivo y un verbo, categorías gramaticales excepcionales en ese listado. Ambas razones harían, en mi opinión, perfectamente prescindible la tilde en esos vocablos. Sabemos que pesa la tradición, pero no le pesó a la RAE aceptar la tendencia del resto de academias de la ASALE que veían totalmente injustificada la tilde de guion o truhan -por citar las abanderadas entre las muchas neomonosílabas a las que afectó este gran acierto: rio, fie, pio, flui, hui, etc.-, y que le llevó, dentro de la lógica de una política lingüística panhispánica, al desarrollo y defensa del concepto de diptongo ortográfico (RAE y ASALE 2005; 2010: 235-236), uno de los conceptos más conseguidos no solo en el camino de la unidad de la lengua, sino también en la coherencia y consistencia de la enseñanza de la ortografía, uno de los principios que, junto con la pronunciación, la etimología y el uso, se ha añadido últimamente en la regulación de las soluciones gráficas para el español. Ya en el siglo XIX Andrés Bello argüía al respecto:

La Academia adoptó tres principios fundamentales para la formación de las reglas ortográficas: pronunciación, uso constante y origen. De estos, el primero es el único esencial y legítimo; la concurrencia de los otros dos es un desorden, que solo la necesidad puede disculpar. La Academia misma, que los admite, manifiesta contradicción en más de una página de su tratado. (ASALE 2010: 22)

Cabría preguntarse, al respecto, qué sucede cuando la pronunciación no puede reflejarse o no quiere prescribirse. Si gran parte de los hablantes del español pronuncian peón y guion uniendo las vocales en una sola sílaba, mientras otros las mantenemos bisílabas, parece lógico buscar una definición de hiato y diptongo que no se atenga a cómo pronunciamos las vocales. Sin necesidad de esgrimir argumentos prohiato o antihiato (Frago 2012: 34), no parece ser función de las reglas de acentuación saber cuántas sílabas tiene vio, dio, lio o rio, sino hacer identificable, de manera unívoca, la vocal tónica de una palabra; esta función prosódica de la tilde es la principal en español (RAE y ASALE 2010: 230), y solo existe una segunda función, la ya comentada diacrítica, que nada tiene que ver al respecto. Sorprende, por tanto, que muchos se resistan al hallazgo, como por ejemplo Manuel Seco: "la Academia está errada y siembra con ello la confusión" (2011: 687), dice. 
En mi opinión, lo que siembra es coherencia. Además, esta regla ni siquiera provoca riesgo de ambigüedad, pues si analizamos cualquiera de estos casos, como puede ser el de la forma guio, es su última vocal la que recibe necesariamente la acentuación prosódica, la acentuemos o no gráficamente: solo puede tratarse del pasado del verbo guiar, dado que el presente lleva obligatoriamente tilde en la i para marcar el hiato.

No obstante, pasó con Bello, pasó con Sarmiento y con otros reformistas no tan radicales, americanos o peninsulares, sus propuestas no fraguaron; muchas de sus causas han sido investigadas recientemente por Juan Antonio Frago Gracia (2012). Hoy en día parece que la política panhispánica de la RAE y de las academias que forman la ASALE tiene en general buena acogida, $y$ por eso pienso que estamos ante un nuevo paradigma que no debe ser menospreciado de cara al potencial de las reformas ortográficas, siempre con la cautela de que el uso deberá refrendarlas.

En segundo lugar, la inconsistencia se fragua entre las diversas obras académicas. Personalmente, esperé con ilusión la 23. ${ }^{a}$ edición del Diccionario de la lengua española (RAE y ASALE 2014), entre otros motivos para dejar de ver, por ejemplo, entradas como "guion $o$ guión", que me desautorizaban como profesora de ortografía y me obligaban a puntualizar, repetidamente, que el diccionario estaba en fase de adaptación a las normas, ya no tan recientes, de 2010. Sin embargo, cuál fue mi decepción cuando encontré soluciones como "sui géneris" o "ex cátedra" [sic], siendo que la Ortografia había sido contundente en las normas que debían regir en el caso de las locuciones latinas: si las escribimos en más de una palabra, deben ser tratadas como las expresiones extranjeras, respetando la grafía de su idioma de origen y marcándolas obligatoriamente en cursiva o entre comillas. Así lo enseñan manuales posteriores que han seguido a las normas de 2010, como el de Gómez Torrego (2011), el del Instituto Cervantes (2012), coordinado por Florentino Paredes, la propia Ortografía básica de la lengua española (RAE y ASALE 2012) u otras propuestas didácticas como la que citamos a continuación:

Latinismos: cuando están adaptados, se acentuarán gráficamente de acuerdo con las reglas generales del español. Ejemplos: accésit, ídem, currículum, réquiem... Cuando forman parte de un nombre científico o de una locución latina, no deben adaptarse: se escriben en cursiva y no llevan tilde. Ejemplo: Laurus nobilis, sui generis y ex cathedra. (Saneleuterio, Rosell y García 2012: 43)

Si bien todo apunta a una rectificación, queremos pensar, sin dejar de exigir su corrección, que se debe a un despiste que sigue la línea de la RAE desde 1870 hasta el Diccionario panhispánico de dudas (RAE y ASALE 2005), 
que propuso un tratamiento específico para las locuciones latinas, que la Ortografía posterior revocó explícitamente (RAE y ASALE 2010: 610). Y queremos pensarlo porque, en efecto, su reforma en 2010 resultó altamente coherente con los principios generales que regulan la ortografía del español.

Hasta aquí, la ambigüedad del título de este artículo se enriquece claramente: la $o$ tiene función semántica disyuntiva, si bien depende del significado que le atribuyamos a "solo en caso de ambigüedad": independientemente de que el primer término lo interpretemos como adjetivo o como adverbio, el enunciado que introduce es susceptible de resultar sugerente. En todo caso, abogo por la coherencia y no temo a la ambigüedad.

\section{TRES RECOMENDACIONES}

Como recapitulación, y en aras de una congruencia que tanto agradecemos -no solo cuando aprendemos, sino también cuando enseñamos la aplicación de una serie de normas, atañan a la disciplina que atañan-, además de la explicación clara y convencida del concepto de diptongo ortográfico ${ }^{5}$, que tantos quebraderos de cabeza ahorra a nuestros estudiantes, recomiendo tres líneas de actuación en cuanto a la acentuación gráfica, las cuales buscan evitar excepciones innecesarias y mejorar la coherencia del sistema, cuestionada en los tres casos expuestos, y las expongo en orden ascendente de contundencia y respaldo normativo:

3.1. Observar con cautela la tilde diacrítica de los monosílabos dé y téltés. No conozco amagos de eliminarla, si bien no me extrañaría que pronto sucediera, conociendo la trayectoria académica, que suele buscar esa coherencia gráfica en el idioma a la que yo apelo. Por mi parte, desde aquí propongo con firmeza que se prescriba que dejen de tildarse, por no cumplir con el requisito de ser

\footnotetext{
5 Trascendiendo sus efectos en la acentuación, el concepto debería implicar actuar con coherencia también en el caso de la conjunción $y$ ante diptongo: al igual que no cambia en $e$ ante palabras como hierro o hielo, tampoco debería hacerlo ante otras como hiato o ion, por mucho que se pronuncien nítidamente como hiatos y así lo recoja la Ortografía (RAE y ASALE 2010: 77).
} 
"ciertos pares de palabras monosílabas y algunas polisílabas de uso muy frecuente" (RAE y ASALE 2010: 231) .

3.2. Tratar las locuciones latinas con los mismos criterios que las expresiones extranjeras, como bien establece la Ortografía académica (RAE y ASALE 2010: 610) y a pesar de que la nueva edición del diccionario académico recoja otras soluciones gráficas. Ello implica escribirlas en cursiva, sin tildes y respetando la grafía latina-verbum gratia: sui generis, post mortem, ex cathedra-, o bien adaptarlas a las reglas españolas convirtiéndolas en préstamos léxicos, cuyo requisito es reducirlas a una sola unidad gráfica, aparte del respeto de las reglas generales de acentuación, de la correspondencia con la pronunciación y de la eliminación de secuencias vocálicas o consonánticas extrañas a nuestro idioma $-v$. $g r .:$ suigéneris, postmórtem, excátedra-.

3.3. No poner el acento gráfico ni al adverbio solo ni a los demostrativos, ni aun en caso de ambigüedad: no solo porque lo permite la Ortografía académica (RAE y ASALE 2010: 269), sino porque auguro que se trata de un período de adaptación para la futura supresión de esta tilde, de la misma manera que en 2010 se acabó con la permisividad establecida en 1999 de tildar palabras como guion o truhan, para hablantes que las pronunciaran nítidamente como bisílabas?

Cabe aludir a las consideraciones de Manuel Seco (2011: 556) cuando se refiere a que "en realidad, la ambigüedad no tiene por qué presentarse nunca, porque el contexto la resuelve en cada caso"; de hecho especifica también que "al ser claramente restrictivo el uso de la tilde en el adverbio solo, lo más recomendable es respetar la norma general de no ponerlo" (Seco 2011: 556).

6 Si también se eliminara el acento gráfico de sé (verbo) y de sí (sustantivo), la tilde diacrítica solo afectaría a pronombres personales ( $t u ́$, él, $m i ́, s i$ ) y adverbios ( $s i$ [a veces sustantivado], más [a veces adjetivado, pronominalizado o conjuntivado]), además del sistema cerrado de los interrogativos y exclamativos.

Esta permisividad se establecía, literalmente, como sigue: "En este caso es admisible el acento gráfico, impuesto por las reglas de ortografía anteriores a estas, si quien escribe percibe nítidamente el hiato y, en consecuencia, considera bisílabas palabras como las mencionadas: fié, huí, riáis, guión, Sión, etc." (RAE 1999: 27). 
Aparte de que como filóloga e investigadora juzgue completamente desafortunado el fragmento citado al principio de esta nota, relativo a la permisión académica de acentuar gráficamente el adverbio solo y los pronombres demostrativos en casos de doble interpretación (RAE y ASALE 2010: 269), como profesora y especialista en didáctica de la ortografía solo -y espero que no sola- puedo recomendar a mis alumnos y alumnas que se acostumbren a jamás tildar estas palabras. Otra cosa es que deba advertirles que, como futuros docentes que son, por ahora no puedan tachar una tilde de estas características cuando corrijan un texto... ¿o sí pueden si no hay peligro de ambigüedad?

\section{REFERENCIAS BIBLIOGRÁFICAS}

Álvarez Mellado, Elena. 2017. "Sólo" y la tilde de la nostalgia. El diario.com, 15 de enero [en línea]. Disponible en https://www.eldiario.es/zonacritica/Solo-tildenostalgia_6_601299895.html [Consulta 10/05/2018].

Asociación de Academias de la Lengua Española (ASALE). 2010. Gramática de la libertad. Andrés Bello y la unidad lingüistica panhispánica. Valparaíso: Aguilar Chilena de Ediciones.

El Mundo. 1996. Libro de estilo. Madrid: Unidad Editorial, 1. ${ }^{\text {a }}$ ed.

Frago Gracia, Juan Antonio. 2012. Razones de las reformas ortográficas en la América independiente y causas de su fracaso. Boletín de Filología 47 (2): 11-46 [en línea]. Disponible en http://www.boletinfilologia.uchile.cl/index.php/BDF/article/ view/26564/28016 [Consulta 25/03/2016].

Gándara, Yolanda. 2014. ¿Quién va ganando en lo de la tilde de "solo"? Jot Down [en línea]. Disponible en http://www.jotdown.es/2014/02/quien-va-ganando-en-lo-de-la-tilde-desolo/ [Consulta 10/05/2018].

Gómez Torrego, Leonardo. 2011. Las normas académicas: últimos cambios. Madrid: Ediciones SM-FSM.

Instituto CeRVANTES. 2012. El libro del español correcto: claves para hablar y escribir bien en español (Florentino Paredes García, Salvador Álvaro García, Zaida Núñez Bayo, Luna Paredes Zurdo). Barcelona: Espasa.

Real Academia Española (RAE). 1726-1739/1969. Diccionario de autoridades. Madrid: Gredos.

1857. Prontuario de ortografía de la lengua castellana para el uso de las escuelas públicas. Madrid: Imprenta Nacional.

1999. Ortografia de la lengua española. Madrid: Espasa Calpe.

Real Academia Española (RAE) y Asociación de Academias de la Lengua Española (ASALE). 2005. Diccionario panhispánico de dudas. Madrid: Santillana [en línea]. Disponible en dpd.rae.es [Consulta 25/03/2016].

2010. Ortografía de la lengua española. Madrid: Espasa.

2012. Ortografía básica de la lengua española. Barcelona: Espasa Libros. 
2014. Diccionario de la lengua española. Madrid: Espasa, 2 vols. 23. ${ }^{a}$ edición [en línea]. Disponible en dle.rae.es [Consulta 25/03/2016].

Saneleuterio, Elia, Maria Rosell y Gloria García. 2012. Trabajo cooperativo para el aprendizaje de las reglas ortográficas. Madrid: CEU Ediciones.

Seco, Manuel. 2011. Nuevo diccionario de dudas y dificultades de la lengua española. Barcelona: Espasa Libros. 